Rechtsmedizin $2022 \cdot 32: 212-216$

https://doi.org/10.1007/s00194-021-00525-1

Angenommen: 19. Juni 2021

Online publiziert: 30. August 2021

๑) Der/die Autor(en) 2021

\section{Kombinierter Suizid: Methode nach Kusch und Kopfschuss - ein Fallbericht}

\author{
A. Weber ${ }^{1} \cdot$ W. Rabl ${ }^{2}$ \\ ${ }^{1}$ Universitätsklinik für Neurologie, Medizinische Universität Innsbruck, Innsbruck, Österreich \\ ${ }^{2}$ Institut für Gerichtliche Medizin, Medizinische Universität Innsbruck, Innsbruck, Österreich
}

\title{
Zusammenfassung
}

Die gleichzeitige Anwendung mehrerer Methoden beim Suizid wird als „kombinierter Suizid" bezeichnet. Vorgestellt wird ein Fall, bei dem zunächst alles auf einen Suizid mittels Schusswaffe hindeutet. Die chemisch-toxikologische Analyse ergibt den Nachweis von Benzodiazepinen und einer potenziell letalen Chloroquinkonzentration im Blutserum, was als Methode nach Kusch bezeichnet wird und mit einer Handlungsunfähigkeit einhergehen kann. Die Kombination von Chloroquin mit Diazepam kann aber, in Abhängigkeit von der Dosierung, auch dazu führen, dass die Handlungsfähigkeit erhalten bleibt und auch hohe Chloroquinkonzentrationen überlebt werden. Der vorgestellte Fall soll die potenziellen Wechselwirkungen dieser Wirkstoffe verdeutlichen.

\section{Schlüsselwörter}

Suizidale Schussbeibringung · Diazepam · Chloroquin · Toxikologie · Wechselwirkungen

2018 nahmen sich in Österreich 1209 Menschen selbst das Leben [1]. Bei Suiziden kommt entweder eine Suizidmethode zur Anwendung, oder es werden mehrere Methoden angewendet, was als „kombinierter Suizid“ bezeichnet wird. Die Kombination erfolgt dabei entweder primär geplant oder ergibt sich erst aus dem Handlungsablauf.

Pawellek berichtet in einer Fallstudie von 173 Suiziden, wobei bei $95 \%$ zwei Suizidmethoden zum Einsatz kamen. Die dominierenden Methoden waren dabei Sturz aus großer Höhe, Ertrinken oder Erhängen jeweils in Kombination mit einer Medikamentenintoxikation. Eine eher untergeordnete Rolle spielte in diesem Kollektiv die Beibringung einer Kopfschussverletzung in Kombination mit einer Medikamentenintoxikation (5 von 173 Fällen). Generell lag die Zahl der Fälle mit Beeinträchtigung durch Arzneimittel bei $62 \%$, wobei der Großteil auf Wirkstoffe aus der Gruppe der Benzodiazepine zurückzuführen war. Dabei handelt es sich um Hypnotika, die anxiolytisch, sedativ-hypnotisch, muskel- relaxierend, antikonvulsiv sowie amnesiogen wirken [6].

Prinzipiell soll die Kombination von 2 oder mehr Methoden den sicheren Todeseintritt gewährleisten, beschleunigen und/oder schmerzfreier gestalten. Bei der Untersuchung solcher Todesfälle müssen die zeitliche Abfolge der Suizidmethoden und die Frage der evtl. beeinträchtigten Handlungsfähigkeit nach Einsatz der ersten Suizidmethode besonders beachtet werden [12].

\section{Fallbericht}

Vorgestellt wird der Fall eines 44 Jahre alt gewordenen Mannes, bei dem zunächst alles auf einen Suizid mittels Kopfschuss hindeutet. Aufgrund der Gegebenheiten am Vorfallsort kann ein Fremdverschulden nicht ausgeschlossen werden, daher wird seitens der Staatsanwaltschaft eine Obduktion angeordnet. 


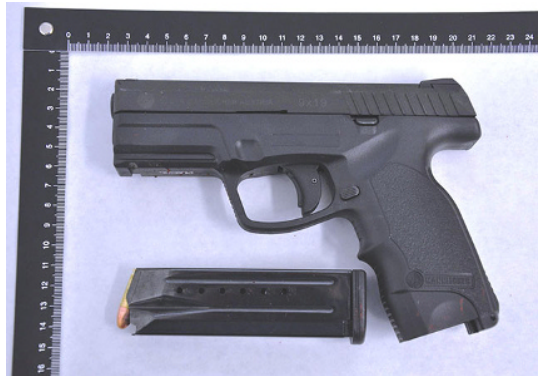

Abb. $1 \Delta$ Am Vorfallsort sichergestellte Schusswaffe

\section{Einleitung}

Der Leichnam wurde vollständig bekleidet und sitzend auf einem Klappstuhl auf einer ebenerdig zugänglichen Terrasse vorgefunden. Er wies eine blutende Verletzung am Hinterkopf auf. Links neben dem Leichnam lag eine Pistole Fabrikat Steyr M-A1 Kaliber $9 \mathrm{~mm}$ (-Abb. 1). Am Vorfallsort zeigten sich keine Einbruchsspuren oder Spuren eines Kampfes.

\section{Methodik}

\section{Ergebnisse der Obduktion}

Die Leichenflecke waren vermindert ausgebildet. Am hohen Hinterkopf zeigte sich ein Ausschuss in Form einer sternförmigen Verletzung der Kopfschwarte (• Abb. 2). An der Oberlippeninnenseite und am Lippenbändchen fanden sich kleinfleckige Einblutungen. Im Mund befand sich ein Blutgerinnsel. Der harte Gaumen wies einen lochartigen Defekt, umgeben von dunklen Einsprengungen, auf. Sonstige frische Verletzungen lagen nicht vor. An der rechten Hand befanden sich zwischen Daumen und Zeigefinger Blutauflagerungen, sowie „Backspatter"-Spritzspuren, die von körpernah nach körperfern verliefen. Am linken Handrücken lagen verwaschene Blutantragungen sowie am Daumenballen punktförmige Antragungen.

In der hohen Hinterhauptsregion bestand ein Schädelbruch mit nach außen abgesprengten Knochenanteilen (• Abb. 3). Das Hirngewebe war gemäß einem Schusskanal vom Gaumen zum Hinterkopf verlaufend aufgepflügt (-Abb.4). Die Sondierung des Schusskanals ergab einen Winkel von ca. $50-55^{\circ} \mathrm{C}$ von vorne nach hinten ansteigend. In Speise- und Luftröhre befand

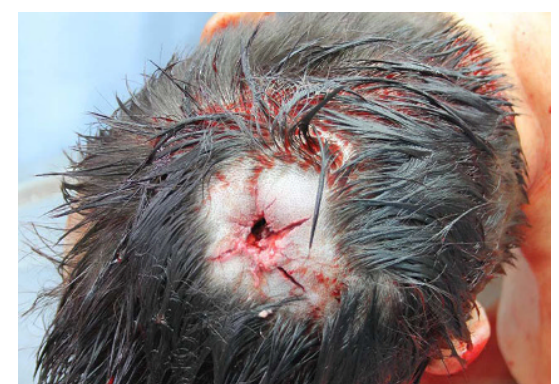

Abb. $2 \Delta$ Sternförmige Ausschusswunde am Hinterkopf

sich reichlich Blut; die Hauptbronchien waren blutgefüllt. Die Luftembolieprobe beim Eröffnen der rechten Herzhälfte verlief positiv. An der Lunge ergaben sich Befunde einer Blutaspiration. Der Mageninhalt bestand aus etwa $20 \mathrm{ml}$ schleimiger, gelbgrünlicher Flüssigkeit ohne offensichtliche Tablettenrückstände.

Die Befunde waren mit einem suizidalen Mundschuss zwanglos in Einklang zu bringen.

\section{Forensisch-toxikologische Analytik}

Zur toxikologischen Analyse wurden im Rahmen der Obduktion peripheres Blut, Harn sowie Galle asserviert. Das Blut wurde im Zuge der Probenvorbereitung mit einem internen Standard versetzt, zentrifugiert und einer Festphasenextraktion unterzogen. Die rekonstituierten Proben wurden anschließend flüssigchromatographisch (MS/MS-Detektion) sowie gaschromatographisch-massenspektrometrisch einer quantitativen Analyse unterzogen. Die Harnproben wurden underivatisiert und acetyliert gas- und flüssigchromatographisch-massenspektrometrisch qualitativ analysiert. Auf eine toxikologische Untersuchung der Galle wurde primär verzichtet.

\section{Ergebnisse}

Im Rahmen der flüssigchromatographischen Untersuchung des Serums wurden die Schlaf- und Beruhigungsmittel, aus der Gruppe der Benzodiazepine, Diazepam $(118 \mu \mathrm{g} / \mathrm{l})$ sowie dessen Stoffwechselprodukt Nordazepam $(31 \mu \mathrm{g} / \mathrm{l})$ vorgefunden. Beide Konzentrationen lagen unter dem therapeutischen Bereich [9]. Gaschromatographisch wurden die Medikamenten-

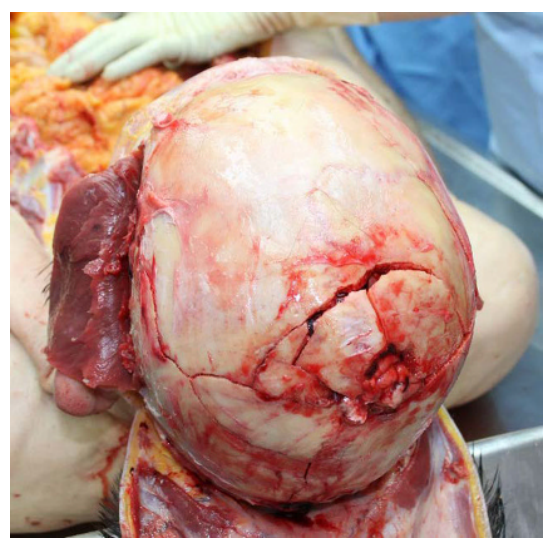

Abb. $3 \Delta$ Schädelbruchlinien in der hohen Hinterhauptsregion mit nach außen abgesprengten Knochenanteilen

wirkstoffe Tramadol (Schmerzmittel aus der Gruppe der Opioide, $400 \mu \mathrm{g} / \mathrm{l}$, therapeutischer Bereich[18]), Metoclopramid (Antiemetikum, $100 \mu \mathrm{g} / \mathrm{l}$, therapeutischer Bereich [19]) und Chloroquin $(9900 \mu \mathrm{g} / \mathrm{l}$, hochtoxischer Bereich [8]) nachgewiesen. Bei der Untersuchung der Harnprobe auf organische Wirkstoffe wurden qualitativ die Wirkstoffe Tramadol, Chloroquin und Metoclopramid nachgewiesen. Zusätzlich wurden die Wirkstoffe Temazepam, Coffein sowie Cotinin (Nikotinstoffwechselprodukt) detektiert. Die Bestimmungen von Blut- sowie Harnalkoholkonzentration ergaben jeweils $0,00 \%$.

\section{Diskussion}

Als Todesursache wird eine Kombination aus hohem Blutverlust mit einer venösen Luftembolie infolge des Mundschusses angenommen. Die hochtoxische, potenziell letale Serumkonzentration von Chloroquin, welche bei der toxikologischen Analyse festgestellt wurde, erhebt aber zusätzlich die Frage einer eingeschränkten, möglicherweise sogar aufgehobenen Handlungsfähigkeit. Tramadol und Benzodiazepine verstärken sich zudem gegenseitig in ihrer zentral dämpfenden Wirkung.

Die nachgewiesene Kombination der Wirkstoffe Chloroquin und Diazepam entspricht der "Methode nach Kusch". Auch der Sterbehelfer Peter Puppe gibt in seinen Büchern diese Arzneimittelkombination als Methode für einen selbstbestimmten Tod an. Zusätzlich führt er in seiner Anleitung auch das Antiemetikum Metoclo- 


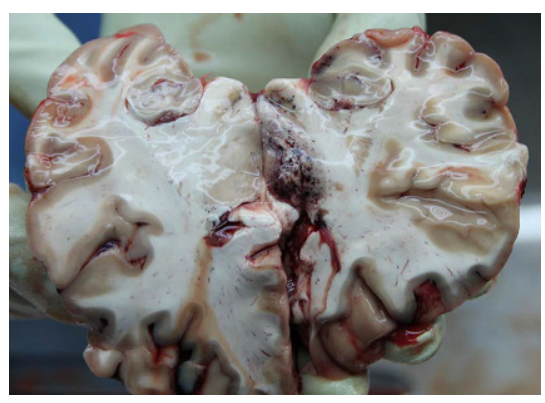

Abb. $4 \Delta$ Aufgepflügtes Hirngewebe gemäß einem Schusskanal, vom Gaumen in Richtung Hinterhaupt verlaufend

pramid zur "Vorbereitung des Magens" an [13]. Auch dieses Medikament konnte bei dem vorgestellten Fall im Blutserum nachgewiesen werden. Aus den Ermittlungen der Polizei ging hervor, dass eine Internetrecherche zur tödlichen Dosis von Chloroquin und Diazepam am Laptop des Verstorbenen vorgenommen wurde. Es ist also denkbar, dass diese Suizidmethode in Betracht gezogen wurde bzw. gezielt nach einer Anleitung dazu gesucht wurde.

Aufgrund der langen Halbwertszeit von Chloroquin (1 bis 2 Wochen [7]) und Diazepam (> 20h [6]) wäre es grundsätzlich auch denkbar, dass die Einnahme der medikamentösen Wirkstoffe bereits einige Zeit vor dem Todeseintritt nicht zum "Erfolg“ geführt hat und es daher zum Gebrauch der Schusswaffe kam. Das Konzentrationsverhältnis zwischen Diazepam und Nordazepam deutet allerdings auf einen Konsum in näherem zeitlichem Zusammenhang zum Todeseintritt hin. Auch die therapeutische Konzentration von Metoclopramid im Blutserum, welches eine Halbwertszeit von wenigen Stunden aufweist, ist ein Indiz für einen todeszeitnäheren Medikamentenkonsum (außer dieses Medikament wurde zu einem späteren Zeitpunkt nochmals einzeln eingenommen) [6]. Es ist also wahrscheinlicher, dass diese beiden Methoden bewusst kombiniert wurden, um den Todeseintritt „abzusichern".

Damit erhebt sich nach wie vor die Frage, ob der Mann in der Lage war selbst den tödlichen Schuss abzugeben, nachdem er eine potenziell letale Dosis Chloroquin in Kombination mit Diazepam eingenommen hatte. Folgt man der Anleitung von Puppe, dann solle diese Kombination innerhalb von wenigen Minuten bzw. schlagartig zur Bewusstlosigkeit führen [14].

Gleichwohl kann genau in dieser eingenommenen Wirkstoffkombination der Grund dafür liegen, dass eine Handlungsfähigkeit u.U. eben doch erhalten bleibt. Denn die kombinierte Einnahme dieser beiden Substanzen für einen Suizid erscheint widersprüchlich, da es einige Publikationen gibt, welchen zu entnehmen ist, dass eine akute Chloroquinintoxikation mit Diazepam erfolgreich behandelt werden kann [3, 15, 17, 21]. Diese kurative Wirkung von Diazepam scheint jedoch dosisabhängig zu sein. So beschreiben Crouzette et al. in-vivoExperimente mit Ratten, die mit Chloroquin vergiftet und mit unterschiedlichen Konzentrationen von Diazepam behandelt wurden. Den Tieren wurden $330 \mathrm{mg} / \mathrm{kgKG}$ Chloroquin (entsprechend der LD 50) verabreicht, und anschließend wurden entweder $2,5 \mathrm{mg}, 7,5 \mathrm{mg}, 20 \mathrm{mg}, 50 \mathrm{mg}$ oder $148 \mathrm{mg}$ Diazepam intraperitoneal appliziert. Dabei konnte festgestellt werden, dass die Mortalität bei Gabe von $20 \mathrm{mg} /$ kgKG Diazepam am niedrigsten war [4]. Auch in der Notfallbehandlung von Chloroquinvergiftungen wird Diazepam als wichtiges „Antidot" beschrieben [15].

Chloroquin wird sowohl zur Malariaprophylaxe wie auch zur Behandlung von rheumatischen Erkrankungen, wie beispielsweise Lupus erythematodes, eingesetzt. Im Zuge des Einsatzes von Chloroquin für diese Erkrankungen kam es immer wieder zum Auftreten von kardialen Nebenwirkungen. Es wurden beispielsweise Fälle von QT-Zeit-Verlängerungen beschrieben, welche zu einer lebensgefährlichen Arrhythmie des Herzens führen können [2, 10, 11]. Im Gegensatz dazu werden Diazepam kardioprotektive Eigenschaften zugesprochen. Shackebaei et al. beschreiben die Behandlung von hyperthyreotischen, ischämischen Rattenherzen mit unterschiedlichen Konzentrationen von Diazepam. Für die Einschätzung des myokardialen Schadens und der Erholungsrate nach 40-minütiger Ischämie mit anschließender 45minütiger Reperfusion wurden folgende kardiale Parameter erfasst: linksventrikulärer Druck, Herzfrequenz, Flussrate der Herzkranzgefäße sowie das Druckratenprodukt. Die Ergebnisse zeigten, dass sich hyperthyreotische Rattenherzen schlechter erholten als Herzen der Kontrollgruppe. Eine zusätzliche Gabe von $1 \mathrm{mg} /$ kgKG Diazepam führte allerdings zu einer vergleichbar guten Erholung wie in der Kontrollgruppe. Vermutet wird dabei, dass Diazepam als Phosphodiesterase-4-Inhibitor fungiert [16]. Phosphodiesterasen kommen in unterschiedlichen Geweben im Körper vor und spielen eine Rolle im Zellstoffwechsel sowie bei entzündlichen Prozessen. Phosphodiesterase-Inhibitoren wirken entzündungshemmend und kommen beispielsweise bei Erkrankungen wie COPD oder Asthma bronchiale zum Einsatz [20].

Die Inhibierung von Phosphodiesterase-4 führt zur Blockade der Genexpression von Tumor-Nekrose-Faktor a (TNFa). Shackebaei et al. vermuten, dass die Reduktion des TNF-a-Proteins das Herz vor einem Reperfusionsschaden schützt [16]. Bei einer akuten myokardialen Ischämie kann es zu einer Verlängerung des QTIntervalls kommen, dasselbe gilt für eine Chloroquinintoxikation. Daher könnte der protektive Effekt von Diazepam auch auf dem oben beschriebenen Effekt beruhen. Wie bei Shackebaei et al. beschrieben, hat dabei eine Dosis von $1 \mathrm{mg} / \mathrm{kgKG}$ den besten Effekt. Bei einer $80 \mathrm{~kg}$ schweren Person würde das $80 \mathrm{mg}$ Diazepam bedeuten, was allerdings stark von der für einen Suizid vorgeschlagenen Dosis von 500-1000 mg abweicht.

Auch gibt es Fallberichte, bei denen es wider Erwarten nicht zu einer Sedierung durch hohe Dosen Diazepam kommt, wenn der Wirkstoff in Kombination mit Chloroquin eingenommen wird, was die Wechselwirkung beider Substanzen verdeutlicht. Croes et al. berichten von einer Frau mit akuter Chloroquinüberdosis (Serumkonzentration $7870 \mu \mathrm{g} / \mathrm{l}$ ), die mit $2 \mathrm{mg} / \mathrm{kgKG}$ Diazepam behandelt wurde. Entgegen der Erwartung blieb die Frau trotz einer hohen Plasmakonzentration von Diazepam und Nordazepam bei Bewusstsein. Die Autoren vermuten dabei einen antagonistischen Effekt von Chloroquin auf die sedierende Wirkung von Benzodiazepinen [3]. Auch Djelardje berichtet über einen Fall, bei dem ein Mann $5 \mathrm{~g}$ Chloroquin in Kombination mit $500 \mathrm{mg}$ Diazepam eingenommen hat und keinerlei Symptomatik gezeigt habe [5]. 
Für den vorgestellten Fall ist es damit plausibel, dass durch die Wechselwirkungen von Chloroquin und Diazepam die Handlungsfähigkeit und damit die Möglichkeit zur Schussabgabe erhalten blieben.

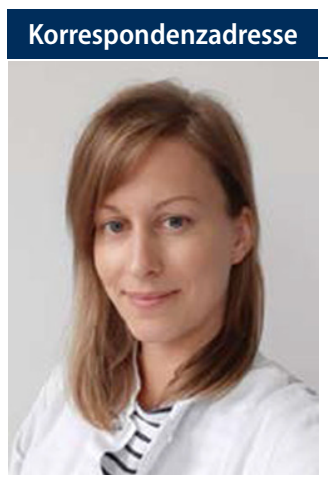

\section{A. Weber, MSc, PhD}

Universitätsklinik für Neurologie, Medizinische Universität Innsbruck

Anichstraße 35, 6020 Innsbruck, Österreich anja.weber@i-med.ac.at

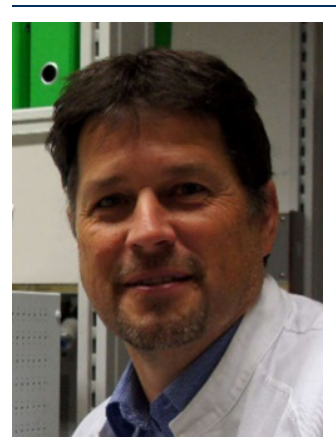

A.Univ.Prof. Dr.med. W. Rabl, MME (Bern) Institut für Gerichtliche Medizin, Medizinische Universität Innsbruck Müllerstraße 44, 6020 Innsbruck, Österreich walter.rabl@i-med.ac.at

Funding. Open access funding provided by University of Innsbruck and Medical University of Innsbruck.

\section{Einhaltung ethischer Richtlinien}

Interessenkonflikt. A. Weber und W. Rabl geben an, dass kein Interessenkonflikt besteht.

Für diesen Beitrag wurden von den Autoren keine Studien an Menschen oder Tieren durchgeführt. Für die aufgeführten Studien gelten die jeweils dort angegebenen ethischen Richtlinien.

Open Access. Dieser Artikel wird unter der Creative Commons Namensnennung 4.0 International Lizenz veröffentlicht, welche die Nutzung, Vervielfältigung, Bearbeitung, Verbreitung und Wiedergabe in jeglichem Medium und Format erlaubt, sofern Sie den/die

\section{Combined suicide: method of Kusch and gunshot to the head - a case report}

The use of more than one method for committing suicide is known under the term "combined suicide". A case is presented in which the initial findings were indicative for a suicide using a shotgun. In contrast, the toxicological analysis detected benzodiazepines and a potentially fatal concentration of chloroquine in blood serum, which is described as the method of Kusch and could be in line with the inability to fire a shotgun. Despite this, under specific circumstances the combination of chloroquine with diazepam could lead to a retained ability to act and to survive even critical doses of chloroquine. This case report outlines the potential interaction of these drugs.

\section{Keywords}

Suicidal gunshot · Diazepam · Chloroquine $\cdot$ Toxicology $\cdot$ Drug interaction

ursprünglichen Autor(en) und die Quelle ordnungsgemäßnennen, einen Link zur Creative Commons Lizenz beifügen und angeben, ob Änderungen vorgenommen wurden.

Die in diesem Artikel enthaltenen Bilder und sonstiges Drittmaterial unterliegen ebenfalls der genannten Creative Commons Lizenz, sofern sich aus der Abbildungslegende nichts anderes ergibt. Sofern das betreffende Material nicht unter der genannten Creative Commons Lizenz steht und die betreffende Handlung nicht nach gesetzlichen Vorschriften erlaubt ist, ist für die oben aufgeführten Weiterverwendungen des Materials die Einwilligung des jeweiligen Rechteinhabers einzuholen.

Weitere Details zur Lizenz entnehmen Sie bitte der Lizenzinformation auf http://creativecommons.org/ licenses/by/4.0/deed.de.

\section{Literatur}

1. Bundesministerium Arbeit, Soziales, Gesundheit, Pflege und Konsumentenschutz (2019) Broschüre Suizid und Suizidprävention in Österreich. http://www.kriseninterventionszentrum.at/wpcontent/uploads/2020/06/Suizidbericht-2019. pdf.Zugegriffen: 14.Jun.2021

2. Chen CY, Wang FL, Lin CC (2006) Chronic hydroxychloroquine use associated with QT prolongation and refractory ventricular arrhythmia. Clin Toxicol 44:173-175

3. Croes K, Augustijns P, Sabbe M et al (1993) Diminished sedation during diazepam treatment for chloroquine intoxication. Pharm World Sci 15:83-85

4. Crouzette J, Vicaut E, Palombo S et al (1983) Experimental assessment of the protective activity of diazepam on the acute toxicity of chloroquine. J Toxicol Clin Toxicol 20:271-279

5. Djelardje S (1976) In: Abidjan TMU (Hrsg) Intoxications volontairs par la chloroquine These Medicale

6. Eckard O, Eberhard H, Klaus K (2002) Pharmakologie und Toxikologie, 3. Aufl. Springer, Berlin Heidelberg, S122

7. Estler C, Schmidt H (2006) Pharmakologie und Toxikologie für Studium und Praxis. Schattauer, Stuttgart

8. Imd Labor Berlin (2021) Hydroxychloroquin. https://www.imd-berlin.de/leistungsverzeichnis/ parameter.html?tx_ajdiagnostics_analyse $\% 5$ Banalyse $\% 5 \mathrm{D}=150438 \&$ tx_ajdiagnostics_ analyse $\% 5$ Btitle $\% 5 \mathrm{D}=$ Hydroxychloroquin\&tx ajdiagnostics_analyse $\% 5$ Bsynonym $\% 5 \mathrm{D}=$ Medika mente\&tx_ajdiagnostics_analyse $\% 5$ Baction $\% 5 D$ $=$ showmod\&tx_ajdiagnostics_analyse $\% 5$ Bcontrol ler\%5D=Analyse \&cHash=6db19bbb913f3deec444 312d082c3875. Zugegriffen 14.Jun.2021

9. Möller HJ, Laux G, Kapfhammer HP (2008) Psychiatrie und Psychotherapie. Band 1 Allgemeine Psychiatrie, 3. Aufl. Springer, Berlin Heidelberg, S 174

10. Morgan ND, Patel SV, Dvorkina O (2013) Suspected hydroxychloroquine-associated QT-interval prolongation in a patient with systemic lupus erythematosus. J Clin Rheumatol 19:286-288

11. O'laughlin JP, Mehta PH, Wong BC (2016) Life threatening severe QTC prolongation in patient with systemic lupus erythematosus due to hydroxychloroquine. Case Rep Cardiol 2016:4626279

12. Pawellek S (2013) Phänomenologie und statistische Auswertung kombinierter Suizide und suizidaler Mehrfachschädigungen - Eine retrospektive Studie aus dem Institut für Rechtsmedizin derCharité-Universitätsmedizin Berlin im 10- Jahres - Zeitraum von 1997-2006. https://refubium. fu-berlin.de/bitstream/handle/fub188/12700/ diss_s.pawellek.pdf?sequence $=1$ \&isAllowed $=y$. Zugegriffen 11.Jun.2021

13. Puppe $P$ (2014) Sterbehilfe 4+1; Wege zum selbstbestimmten Sterben. epubli $\mathrm{GmbH}$, Berlin, S71

14. Puppe $P$ (2019) Suizidhilfe und Methoden - Der sanfte Tod 2019. epubli GmbH, Berlin

15. Schaper A, Ebbecke M (2017) Intox, detox, antidotes - Evidence based diagnosis and treatment of acute intoxications. Eur J Intern Med 45:66-70

16. Shackebaei D, Asadmobini A, Hesari Met al (2012) Cardioprotective effect of diazepam on ischemiareperfused isolated hyperthyroid rat heart. Turk J Biol 36:598-605

17. StiffG, Robinson D, Cugnoni HLet al (1991) Massive chloroquine overdose-a survivor. Postgrad Med J 67:678-679

18. Universitätsklinik Heidelberg (2021) Tramadol. https://www.klinikum.uni-heidelberg.de/suche? $q=$ tramadol.Zugegriffen 14.Jun.2021

19. Universitätsklinikum Heidelberg (2021) Metoclopramid. https://www.klinikum.uni-heidelberg. de/suche?tx_solr\%5Bq\%5D=metoclopramid. Zugegriffen 14.Jun.2021 


\title{
In eigener Sache
}

20. Wikipedia (2020) PDE-4-Hemmer. https:// de.wikipedia.org/w/index.php?title=PDE4-Hemmer\&oldid=204835078. Zugegriffen 14.Jun. 2021

21. Yanturali S (2004) Diazepam for treatment of massive chloroquine intoxication. Resuscitation. https://doi.org/10.1016/j.resuscitation.2004.06. 018

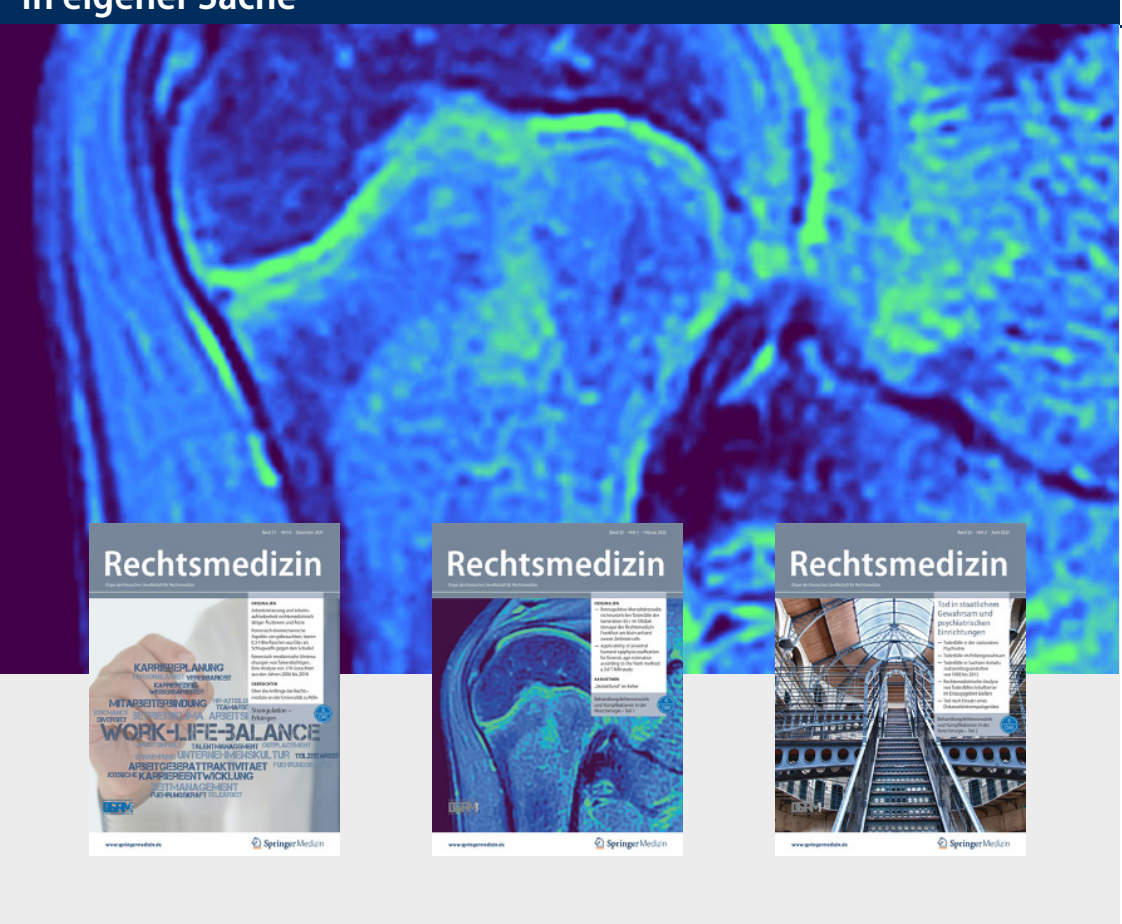

\section{Leitthemenübersicht Rechtsmedizin 2022}

Die Zeitschrift Rechtsmedizin bietet Ihnen jeden zweiten Monat umfassende und aktuelle Beiträge zu interessanten Themenschwerpunkten aus allen Bereichen der Rechtsmedizin.

$\begin{array}{ll}-01 / 2022 & \text { Varia } \\ -02 / 2022 & \text { Tod in staatlichem Gewahrsam } \\ -03 / 2022 & \text { Varia } \\ -04 / 2022 & \text { Kongressheft } \\ -05 / 2022 & \text { Varia } \\ -06 / 2022 & \text { Varia }\end{array}$

Alle Inhalte von Rechtsmedizin finden Sie unter www.springermedizin.de/rechtsmedizin.

SpringerMedizin.de bietet Ihnen Zugang zu allen elektronisch verfügbaren Ausgaben Ihrer Zeitschrift - unabhängig vom Beginn Ihres Abonnements.

Möchten Sie ein bereits erschienenes Heft nachbestellen? Einzelne Ausgaben können Sie direkt bei unserem Kundenservice zum Preis von je EUR 94,- zzgl. Versandkosten beziehen.

\author{
So erreichen Sie unseren Kundenservice: \\ Springer Customer Service Center GmbH \\ Kundenservice Zeitschriften \\ Tiergartenstr. 15, 69126 Heidelberg \\ Tel.: +49 6221 345-4303 \\ Fax: $+496221345-4229$ \\ E-Mail: leserservice@springer.com
}

